\title{
Effect of sulodexide on endothelial glycocalyx and vascular permeability in patients with type 2 diabetes mellitus
}

\author{
L. N. Broekhuizen • B. A. Lemkes • H. L. Mooij • M. C. Meuwese • H. Verberne • \\ F. Holleman • R. O. Schlingemann • M. Nieuwdorp • E. S. G. Stroes • H. Vink
}

Received: 16 June 2010 /Accepted: 16 August 2010 /Published online: 25 September 2010

(C) The Author(s) 2010. This article is published with open access at Springerlink.com

\begin{abstract}
Aims/hypothesis Endothelial glycocalyx perturbation contributes to increased vascular permeability. In the present study we set out to evaluate whether: (1) glycocalyx is perturbed in individuals with type 2 diabetes mellitus, and (2) oral glycocalyx precursor treatment improves glycocalyx properties.

Methods Male participants with type 2 diabetes $(n=10)$ and controls $(n=10)$ were evaluated before and after 2 months of sulodexide administration ( $200 \mathrm{mg} /$ day). The glycocalyx dimension was estimated in two different vascular beds using sidestream dark field imaging and combined fluorescein/indocyanine green angiography for sublingual and
\end{abstract}

L. N. Broekhuizen and B. A. Lemkes contributed equally to this study.

L. N. Broekhuizen · H. L. Mooij • M. C. Meuwese •

M. Nieuwdorp $\cdot$ E. S. G. Stroes $(\bowtie) \cdot H$. Vink

Department of Vascular Medicine, Academic Medical Centre,

Room F4.211, Meibergdreef 9,

1105 AZ Amsterdam, the Netherlands

e-mail: e.s.stroes@amc.uva.nl

B. A. Lemkes · F. Holleman • M. Nieuwdorp

Department of Internal Medicine, Academic Medical Centre,

Amsterdam, the Netherlands

H. Verberne

Department of Nuclear Medicine, Academic Medical Centre,

Amsterdam, the Netherlands

R. O. Schlingemann

Netherlands Institute for Neuroscience,

Amsterdam, the Netherlands

H. Vink

Department of Physiology, Cardiovascular Research Institute Maastricht, Maastricht University,

Maastricht, the Netherlands retinal vessels, respectively. Transcapillary escape rate of albumin $\left(\right.$ TER $\left._{\mathrm{alb}}\right)$ and hyaluronan catabolism were assessed as measures of vascular permeability.

Results Both sublingual dimensions $(0.64$ [0.57-0.75] $\mu \mathrm{m}$ vs $0.78[0.71-0.85] \mu \mathrm{m}, p<0.05$, medians [interquartile range]) and retinal glycocalyx dimensions (5.38 [4.886.59] $\mu \mathrm{m}$ vs 8.89 [4.74-11.84] $\mu \mathrm{m}, p<0.05$ ) were reduced in the type 2 diabetes group compared with the controls whereas TER $_{\text {alb }}$ was increased $(5.6 \pm 2.3 \%$ vs $3.7 \pm 1.7 \%$ in the controls, $p<0.05$ ). In line with these findings, markers of hyaluronan catabolism were increased with diabetes (hyaluronan $137 \pm 29$ vs $81 \pm 8 \mathrm{ng} / \mathrm{ml}$ and hyaluronidase $78 \pm 4$ vs $67 \pm 2 \mathrm{U} / \mathrm{ml}$, both $p<0.05$ ). Sulodexide increased both the sublingual and retinal glycocalyx dimensions in participants with diabetes (to $0.93[0.83-0.99] \mu \mathrm{m}$ and to 5.88 [5.33-6.26] $\mu \mathrm{m}$, respectively, $p<0.05)$. In line, a trend towards $\mathrm{TER}_{\mathrm{alb}}$ normalisation (to $4.0 \pm 2.3 \%$ ) and decreases in plasma hyaluronidase (to $72 \pm 2 \mathrm{U} / \mathrm{ml}, p<$ 0.05 ) were observed in the diabetes group.

Conclusion/interpretation Type 2 diabetes is associated with glycocalyx perturbation and increased vascular permeability, which are partially restored following sulodexide administration. Further studies are warranted to determine whether long-term treatment with sulodexide has a beneficial effect on cardiovascular risk.

Trial registration www.trialregister.nl NTR780/http://isrctn. org ISRCTN82695186

Funding An unrestricted Novartis Foundation for Cardiovascular Excellence grant (2006) to M. Nieuwdorp/E. S. G. Stroes, Dutch Heart Foundation (grant number 2005T037)

Keywords Diabetes mellitus type 2 . Endothelial glycocalyx $\cdot$ Hyaluronan $\cdot$ Sulodexide .

Vascular permeability 


$\begin{array}{ll}\text { Abbreviations } \\ \text { ARB } & \text { Angiotensin-receptor blocker } \\ \text { CRP } & \text { C-reactive protein } \\ \text { FAG } & \text { Fluorescein angiography } \\ \text { ICG } & \text { Indocyanine green angiography } \\ \text { GAG } & \text { Glycosaminoglycan } \\ \text { GlcNAc } & N \text {-acetyl-glucosamine } \\ \text { SDF } & \text { Sidestream dark field } \\ \text { TER }_{\text {alb }} & \text { Transcapillary escape rate of albumin }\end{array}$

\section{Introduction}

Diabetes mellitus is characterised by an increased propensity towards vascular complications. Microvascular complications, such as retinopathy and nephropathy, as well as macrovascular complications are largely responsible for morbidity and mortality in type 2 diabetes mellitus patients [1-3]. An early sign of vascular damage is increased vascular permeability, which may eventually be associated with microalbuminuria at a later stage. Although the pathophysiology leading to increased vascular permeability and microalbuminuria and the link with cardiovascular complications have not been fully elucidated, hyperglycaemia is likely to be a causal factor [4].

The endothelial glycocalyx layer consisting of proteoglycans with their associated glycosaminoglycans (GAGs) [5-9], covers the luminal side of each vessel wall. Under physiological conditions, the glycocalyx shields the endothelial lining from direct contact with circulating blood elements [3]. Recently, we observed that acute hyperglycaemia resulted in a profound perturbation of the endothelial glycocalyx, which coincided with vascular dysfunction and activation of the coagulation system in healthy volunteers [5]. In fact, with respect to chronic hyperglycaemia, patients with type 1 diabetes mellitus were characterised by a $50 \%$ decrease in glycocalyx volume. Loss of glycocalyx volume closely correlated with increased plasma hyaluronidase levels, indicative of enhanced hyaluronan catabolism [10]. Glycocalyx perturbation has been associated with a wide spectrum of vascular abnormalities in experimental models, including increased vascular permeability as well as increased adhesion of leucocytes and thrombocytes $[11,12]$. In animal models, restoration of the glycocalyx reversed these abnormalities [11]. This led to the concept that reversal of glycocalyx damage may be an attractive therapeutic target capable of preserving vascular integrity. In vitro studies have suggested that supplementation of glycocalyx constituents in part restored glycocalyx damage through both increased $\mathrm{N}$ acetyl-glucosamine (GlcNAc)-driven GAG synthesis and decreased GAG catabolism $[13,14]$. Sulodexide is a commercially available compound consisting of heparan sulphate $(80 \%)$ and dermatan sulphate $(20 \%)$. Sulodexide is degraded in the digestive tract into GlcNAc building blocks, leading to an increase in the precursors available for GAG synthesis [14, 15]. In vitro studies have shown that sulodexide attenuates hyperglycaemia-associated endothelial permeability in vitro through enhanced GAG synthesis [16], but the effect of sulodexide treatment on diabetes-associated microalbuminuria in vivo is less clear. In the present study, we thus hypothesised that increasing the precursors available for GAG synthesis could improve endothelial glycocalyx properties in type 2 diabetes mellitus patients. To this end, we evaluated the glycocalyx dimension in two different vascular beds before and after oral administration of sulodexide for 8 weeks.

\section{Methods}

Study population In this investigator-initiated study, we enrolled ten non-smoking men with diabetes mellitus type 2 without microalbuminuria (based on $24 \mathrm{~h}$ urine microalbuminuria measurement in the 6 months preceding the study), retinopathy or macrovascular disease (defined as a history of myocardial infarction, stroke, peripheral vascular disease or signs of macrovascular disease at physical examination). All patients used oral antihyperglycaemic medication and patients using antihypertensive medication were excluded from the study. Statins were discontinued at least 4 weeks prior to study initiation. Ten normoglycaemic non-smoking age-matched healthy men served as an agematched control group. Participants were asked to refrain from heavy physical exercise $24 \mathrm{~h}$ prior to the study visit. Alcohol, caffeine and metformin were withheld at least $12 \mathrm{~h}$ before the study. All participants gave written informed consent, and approval was obtained from the internal review board of the Academic Medical Centre. The study was registered in the Netherlands Trial Register (NTR780/ ISRCTN82695186). The study was carried out in accordance with the principles of the Declaration of Helsinki.

Study design In patients and age-matched controls we measured: (1) local sublingual glycocalyx thickness using sidestream dark field (SDF) imaging; (2) retinal glycocalyx thickness using fluorescein and indocyanine green angiography (FAG/ICG); (3) transcapillary escape rate of albumin $\left(\mathrm{TER}_{\mathrm{alb}}\right)$; and (4) circulating plasma levels of hyaluronan and its degrading enzyme hyaluronidase both at baseline and after 8 weeks of sulodexide administration $(200 \mathrm{mg} /$ day; $25 \mathrm{mg} /$ capsule, Alfa Wasserman, Milan, Italy). Sulodexide is a glycosaminoglycan of natural origin extracted from mammalian intestinal mucosa, containing a mixture of $80 \%$ low-molecular-mass heparan sulphate and 
$20 \%$ dermatan sulphate [15]. Blood pressure was measured three times, from which the means of the last two measurements were used as systolic and diastolic blood pressure values.

Endothelial glycocalyx dimension We assessed the endothelial glycocalyx dimension of both the sublingual and the retinal circulations. The determination of the erythrocyteendothelium gap is the gold standard for glycocalyx measurement in vivo [17] as the endothelial glycocalyx allows limited access to erythrocytes. Using this principle, the sublingual glycocalyx dimension was estimated using SDF imaging [17]. Briefly, in each individual approximately 1,000 measurement sites of $10 \mu \mathrm{m}$ in length were marked in sublingual vessels. At each measurement site, multiple estimates of the erythrocyte column width were made by measuring both the median erythrocyte width as well as the 90th percentile of erythrocyte width distribution. For vessels with diameters ranging from 10 to $20 \mu \mathrm{m}$, a functional estimate of glycocalyx dimension was estimated by comparing the 50th percentile of erythrocyte width with the 90th percentile of erythrocyte width (Fig. 1). Based on these estimates, a single median value for glycocalyx dimension was calculated for each individual. The reproducibility of SDF measurements used to estimate glycocalyx dimension is good within our centre, with an intersession coefficient of variation of $5.6 \pm 3.2 \% \quad(n=10$ controls measured on two separate occasions). The same principle can be applied to retinal endothelial glycocalyx measurement with fluorescence angiography. This method uses the intravascular distribution of two different fluorescent tracers: fluorescein and indocyanine green [18]. Comparable with the exclusion of erythrocytes, the endothelial glycocalyx also allows limited access to plasma macromolecules, whereas smaller tracers can readily permeate into the glycocalyx [19]. In the retinal capillaries, fluorescein fills up the entire vascular compartment while indocyanine green binds to albumin and is therefore excluded by the glycocalyx layer. By calculating the difference between the two compartments, the dimension of the glycocalyx layer in retinal vessels can be estimated. Retinal glycocalyx dimensions, represented by the indocyanine green exclusion zone on retinal angiography, were determined in larger retinal vessels (diameter on FAG: $90 \mu \mathrm{m}$ and above), thereby avoiding the heterogeneity of glycocalyx dimensions in smaller vessels.

\section{Transcapillary escape rate of albumin}

Vascular permeability was determined by the transcapillary escape rate of ${ }^{125}$ I-labelled albumin $\left(\right.$ TER $\left._{\text {alb }}\right)[20] .{ }^{125} \mathrm{I}$ -

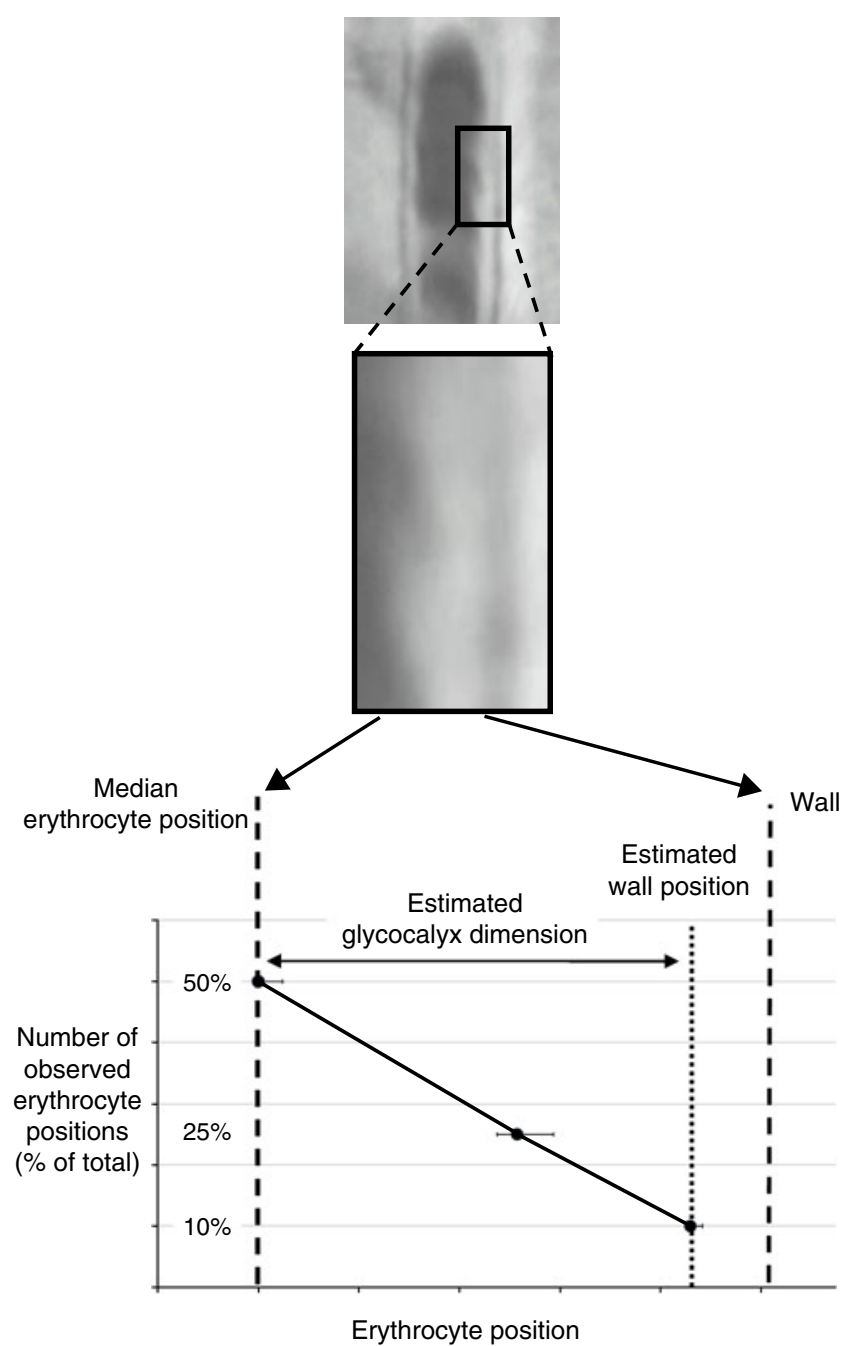

Fig. 1 Measurement of sublingual glycocalyx dimension using SDF imaging

labelled albumin solution of $100 \mathrm{kBq}$ in $5 \mathrm{ml}$ saline was infused as an intravenous bolus. Blood samples were drawn from the contralateral arm at baseline, and at 5, 10, 15, 20, 30,45 and $60 \mathrm{~min}$. Plasma radioactivity was measured in each sample and in a urine sample, collected at the end of the procedure, using a scintillation detector (automatic $\gamma$ counter). TER $\mathrm{R}_{\text {alb }}$ was expressed as the percentage decline in plasma radioactivity from 10 to $60 \mathrm{~min}$ after injection.

\section{Biochemical variables}

Glucose was assessed using the hexokinase method (Glucoquant, Hitachi 917; Hitachi, Valbonne, France). $\mathrm{HbA}_{1 \mathrm{c}}$ was measured by HPLC (Reagens Bio-Rad Laboratories, Veenendaal, the Netherlands) on a Variant II system (Bio-Rad Laboratories, Hemel Hempstead, UK). Plasma C-reactive protein (CRP) levels were measured with a commercially available assay (Roche Diagnostics, Basel, Switzerland). 
Total cholesterol, HDL-C, and triacylglycerols were measured by standard enzymatic methods (Roche Diagnostics). LDL-C was calculated using the Friedewald formula. Alanine aminotransferase and aspartate aminotransferase were measured by pyridoxalphosphate activation assay (Roche Diagnostics). Creatinine was measured by Jaffe kinetic colorimetric test (Roche Diagnostics) on Modular P800 (Roche Diagnostics). For further analysis, plasma aliquots were snap-frozen and stored at $-80^{\circ} \mathrm{C}$. Quantitative total plasma hyaluronan levels were measured by enzymelinked immunosorbent assay (Echelon Biosciences, Salt Lake City, UT, USA) [21]. Plasma hyaluronidase levels were determined with an assay as described previously by Nieuwdorp et al. [10].

\section{Statistical analysis}

Results are expressed as means $\pm \mathrm{SD}$. Baseline differences between controls and patients with type 2 diabetes were tested using an unpaired Mann-Whitney test (two-tailed). Differences within groups before and after treatment were tested using a paired Student's $t$ test (two-tailed). CRP and triacylglycerols were not normally distributed. Therefore, we present medians (interquartile ranges) and used nonparametric tests for these values. Analyses were performed with SPSS version 11.5 (Chicago, IL, USA). A $p$ value $<$ 0.05 was defined as statistically significant.

\section{Results}

Clinical characteristics Clinical characteristics of the participants are listed in Table 1. As compared with controls, patients with type 2 diabetes were characterised by increased glycaemic indices, diastolic blood pressure as well as increased CRP levels. No significant changes in $\mathrm{BMI}, \mathrm{HbA}_{1 \mathrm{c}}$ or glucose levels were observed with sulodexide administration in patients and controls. Other than an increased incidence of epistaxis in one healthy volunteer, no side effects were reported.

Endothelial glycocalyx thickness measured with SDF imaging and FAG/ICG Patients with type 2 diabetes mellitus were characterised by reduced sublingual glycocalyx dimensions compared with the controls at baseline: $0.64(0.57-0.75) \mu \mathrm{m}$ (type 2 diabetes) vs $0.78(0.71-0.85)$ $\mu \mathrm{m}$ (controls; $p<0.05$ type 2 diabetes vs controls; Fig. 2). Following sulodexide treatment the average sublingual glycocalyx thickness increased in diabetic patients (to $0.93[0.83-0.99] \mu \mathrm{m} ; p<0.05$ placebo vs sulodexide; Fig. 3). In controls, sulodexide had no significant effect

Table 1 Baseline characteristics of participants

\begin{tabular}{|c|c|c|c|c|c|c|c|}
\hline \multirow[t]{2}{*}{ Characteristic } & \multicolumn{3}{|c|}{ Participants with type 2 diabetes } & \multicolumn{4}{|l|}{ Controls } \\
\hline & $\begin{array}{l}\text { Baseline } \\
n=10\end{array}$ & $\begin{array}{l}\text { After } 8 \text { weeks } \\
\text { of sulodexide } \\
n=10\end{array}$ & $p^{\mathrm{a}}$ & $\begin{array}{l}\text { Baseline } \\
n=10\end{array}$ & $p^{\mathrm{b}}$ & $\begin{array}{l}\text { After } 8 \text { weeks } \\
\text { of sulodexide } \\
n=10\end{array}$ & $p^{\mathrm{c}}$ \\
\hline Age (years) & $51.9 \pm 8.4$ & $51.9 \pm 8.4$ & $p=\mathrm{NS}$ & $56.3 \pm 8.5$ & $p=\mathrm{NS}$ & $56.3 \pm 8.5$ & $p=\mathrm{NS}$ \\
\hline Duration of diabetes (years) & $5.1 \pm 5.6$ & $5.1 \pm 5.6$ & $p=\mathrm{NS}$ & & & & \\
\hline $\operatorname{BMI}\left(\mathrm{kg} / \mathrm{m}^{2}\right)$ & $29.7 \pm 5.5$ & $29.6 \pm 5.3$ & $p=\mathrm{NS}$ & $27 \pm 2.1$ & $p=\mathrm{NS}$ & $26.9 \pm 1.8$ & $p=\mathrm{NS}$ \\
\hline SBP $(\mathrm{mmHg})$ & $141 \pm 14$ & $139 \pm 18$ & $p=\mathrm{NS}$ & $135 \pm 15$ & $p=\mathrm{NS}$ & $141 \pm 27$ & $p=\mathrm{NS}$ \\
\hline DBP (mmHg) & $87 \pm 8$ & $84 \pm 9$ & $p=\mathrm{NS}$ & $82 \pm 8$ & $p<0.05$ & $81 \pm 6$ & $p=\mathrm{NS}$ \\
\hline Glucose (mmol/l) & $7.1 \pm 2.0$ & $7.3 \pm 2.4$ & $p=\mathrm{NS}$ & $4.9 \pm 0.4$ & $p=0.002$ & $5.2 \pm 0.6$ & $p=\mathrm{NS}$ \\
\hline $\mathrm{HbA}_{1 \mathrm{c}}(\%)$ & $7.1 \pm 1.2$ & $7.1 \pm 1.2$ & $p=\mathrm{NS}$ & $5.6 \pm 0.3$ & $p<0.001$ & $5.5 \pm 0.2$ & $p=\mathrm{NS}$ \\
\hline $\mathrm{TC}(\mathrm{mmol} / \mathrm{l})$ & $5.6 \pm 1.0$ & $5.3 \pm 1.4$ & $p=\mathrm{NS}$ & $6.3 \pm 1.3$ & $p=\mathrm{NS}$ & $5.5 \pm 0.9$ & $p=\mathrm{NS}$ \\
\hline LDL-C (mmol/l) & $3.4 \pm 0.8$ & $3.1 \pm 1.3$ & $p=\mathrm{NS}$ & $4.4 \pm 1.1$ & $p=\mathrm{NS}$ & $3.5 \pm 1.0$ & $p=\mathrm{NS}$ \\
\hline HDL-C (mmol/l) & $1.1 \pm 0.3$ & $1.0 \pm 0.3$ & $p=\mathrm{NS}$ & $1.4 \pm 0.1$ & $p=0.001$ & $1.4 \pm 0.3$ & $p=\mathrm{NS}$ \\
\hline TG (mmol/1) & $2.0(1.4-3.5)$ & $2.1(1.1-3.0)$ & $p=\mathrm{NS}$ & $1.0(0.9-1.3)$ & $p=0.001$ & $1.3(0.8-1.4)$ & $p=\mathrm{NS}$ \\
\hline CRP (mg/l) & $1.9(0.5-5.5)$ & $1.5(0.5-4.4)$ & $p=\mathrm{NS}$ & $1.2(0.5-1.4)$ & $p<0.05$ & $2.6(0.8-14)$ & $p=\mathrm{NS}$ \\
\hline
\end{tabular}

Values expressed as means $\pm \mathrm{SD}$

Triacylglycerol and CRP values are presented as median (interquartile range) due to skewed distribution

DBP, diastolic blood pressure; HR, heart rate; SBP, systolic blood pressure; TC, total cholesterol; TG, triacylglycerols

${ }^{a}$ Diabetes patients at baseline vs diabetes patients after 8 weeks of sulodexide

${ }^{\mathrm{b}}$ Baseline diabetes patients vs baseline controls

${ }^{\mathrm{c}}$ Baseline controls vs control participants after 8 weeks of sulodexide 


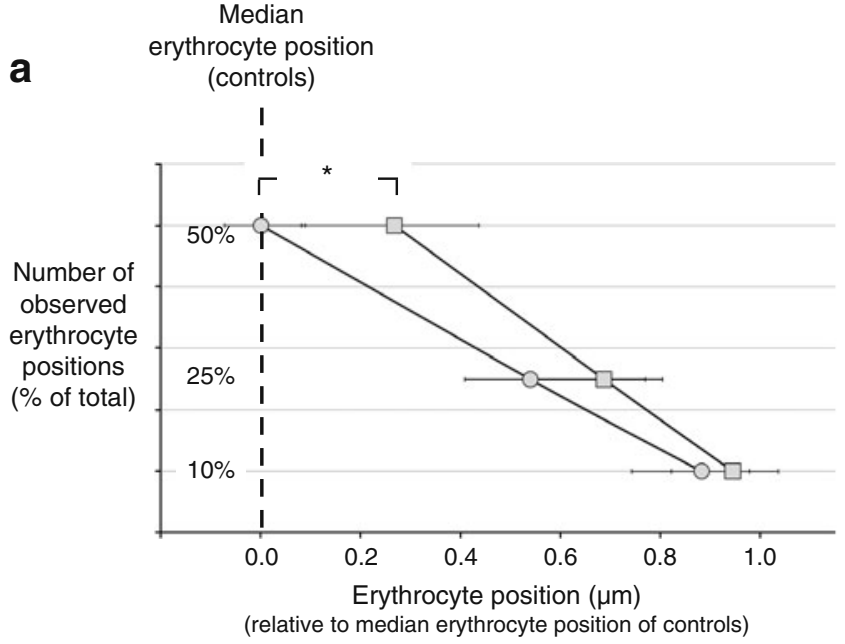

b

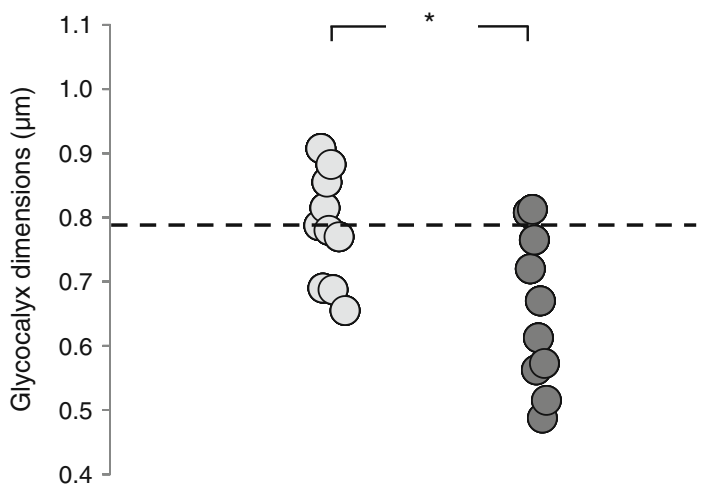

Fig. 2 Endothelial glycocalyx in control and type 2 diabetic participants. a Compared with controls, median erythrocyte width was significantly increased in participants with type 2 diabetes, resulting in a significant movement of erythrocytes towards the vessel wall; $* p<0.05$. Circles, controls; squares, type 2 diabetes group. b Glycocalyx dimensions were reduced in the group with type 2 diabetes because of a significant increase in the median erythrocyte width, while both control and diabetic participants had similar values of the 90th percentiles of the erythrocyte column width (identifying the position of the vessel wall), ${ }^{*} p<0.05$. Light circles, controls; dark circles, type 2 diabetes group

on glycocalyx dimension (increasing to 0.88 [0.80-0.99] $\mu \mathrm{m})$. In a subset of patients and controls ( $n=6$ per group), retinal glycocalyx dimensions were also determined both before and after 8 weeks of sulodexide administration. The retinal glycocalyx dimension was reduced in type 2 diabetes mellitus $(5.38 \mu \mathrm{m}$ [4.88-6.59] vs controls $8.89 \mu \mathrm{m}$ [4.74-11.84], $p<0.05$ type 2 diabetes vs controls; Fig. 4). Following administration of sulodexide for 8 weeks, retinal glycocalyx thickness increased in participants with type 2 diabetes mellitus (5.88 $\mu \mathrm{m}$ [5.33-6.26]; $p=0.05$ vs baseline). In contrast to the effect on sublingual capillary glycocalyx, sulodexide decreased retinal glycocalyx dimension in controls (to 4.87 [3.89-6.33] $\mu \mathrm{m}, p<0.05$ ), particularly in those with a relatively large glycocalyx dimension at baseline (data not shown).
Transcapillary escape rate of albumin $\mathrm{TER}_{\mathrm{alb}}$ in the first hour after infusion was increased in type 2 diabetes mellitus patients $(5.6 \pm 2.3 \%$ in patients vs $3.7 \pm 1.7 \%$ in controls, $p<$ 0.05 ; Fig. 5). Following administration of sulodexide for 2 months, a trend towards normalisation of $\mathrm{TER}_{\mathrm{alb}}$ was observed in those with type 2 diabetes mellitus (to $4.0 \pm$ $2.3 \%, p=0.08)$ whereas in controls $\mathrm{TER}_{\mathrm{alb}}$ was unaffected (to $3.3 \pm 1.6 \%$ ).

Biochemistry Plasma hyaluronan levels were higher in type 2 diabetes mellitus patients compared with healthy controls at baseline ( $137 \pm 29$ vs $81 \pm 8 \mathrm{ng} / \mathrm{ml}, p<0.05$; Fig. 6). Plasma hyaluronidase levels (indicative of both endogenous hyaluronan- and glycocalyx-degrading capacity) were also increased in patients compared with controls $(78 \pm 4$ vs $67 \pm$ $2 \mathrm{U} / \mathrm{ml}, p<0.05$ ), which is in line with data from type 1 diabetes patients $[10,21]$. Following sulodexide administration in participants with type 2 diabetes mellitus, plasma hyaluronidase activity decreased (from $78 \pm 4$ to $72 \pm 2 \mathrm{U} / \mathrm{l}$, $p<0.05)$ whereas hyaluronan levels were comparable $(137 \pm$ 29 vs $129 \pm 15 \mathrm{ng} / \mathrm{ml}$; NS). In the controls, however, hyaluronan increased (from $81 \pm 8$ to $107 \pm 13 \mathrm{ng} / \mathrm{ml}$, $p<0.05$ ) as did plasma hyaluronidase activity (from $67 \pm 2$ to $71 \pm 3 \mathrm{U} / \mathrm{ml}, p<0.05$ ) following sulodexide administration.

\section{Discussion}

In the present study, we show that the endothelial glycocalyx dimensions of both the sublingual and the retinal microcirculation are decreased in patients with type 2 diabetes mellitus compared with healthy controls. Following 8 weeks of sulodexide administration to establish enhanced precursor abundance for GAG synthesis, glycocalyx dimensions improved in both the sublingual and the retinal microvasculature. This improvement coincided with a trend towards improvement of transcapillary albumin escape rate and a reduction in hyaluronan catabolism. These novel findings warrant further studies to evaluate whether endothelial glycocalyx restoration may be of value as an early marker predicting a risk reduction in vascular complication rate in type 2 diabetes mellitus.

Following earlier reports in individuals with type 1 diabetes mellitus, we now demonstrate the presence of glycocalyx perturbation in two different microvascular beds in patients with type 2 diabetes mellitus [10]. The significant reduction in glycocalyx dimensions in both sublingual as well as retinal vessels in type 2 diabetes underpins the systemic nature of glycocalyx perturbation. The latter coincided with a significant increase in the transcapillary escape rate of albumin indicative of increased systemic permeability [21-25], lending further support to 
Fig. 3 The effect of sulodexide on endothelial glycocalyx in control and diabetic participants. a Following sulodexide treatment, median erythrocyte width decreased significantly in the type 2 diabetes group, resulting in a significant restoration of the distance of erythrocytes from the vessel wall; $* p<0.05$. White squares, diabetes group before sulodexide exposure; black squares, diabetes group following sulodexide treatment. b Following sulodexide treatment, median erythrocyte width was not significantly changed in controls, resulting in similar distances of erythrocytes from the vessel. White circles, control group before sulodexide exposure; black circles, diabetes group following sulodexide treatment. c Glycocalyx dimensions at baseline and after 8 weeks of treatment with sulodexide in controls $(n=10$; con and Con Sx, respectively) and diabetic participants ( $n=10$; DM2 and DM2_Sx, respectively) measured by SDF imaging show sublingual glycocalyx dimensions were significantly reduced in type 2 diabetes, and sulodexide treatment restored microvascular glycocalyx dimensions to control values. Individual capillary glycocalyx dimensions were estimated from the transient widening of the erythrocyte column. Data are presented as whisker plots with median (interquartile range). ${ }^{*} p<0.05$

the concept of systemic glycocalyx perturbation in diabetes mellitus. In this respect, experimental studies have implied that the endothelial glycocalyx may be instrumental in maintaining the endothelial barrier function under physiological conditions [11]. Alterations in the proteoglycan/ glycosaminoglycan matrix structure of the endothelial glycocalyx could adversely affect the charge-selective repulsion of negatively charged proteins such as albumin, resulting in increased transvascular leakage in both kidney $[26,27]$ and the systemic vascular barrier in diabetes mellitus [10]. However, systemic albumin clearance (TERalb) may not be the best method to evaluate changes in systemic vascular permeability, particularly in diabetes mellitus, as the impact of impaired reabsorption of albumin in the proximal tubules in diabetes mellitus may outweigh potential changes in systemic albumin leakage, as suggested by Russo et al. [28].

Oral sulodexide supplementation was associated with a significant improvement of both sublingual and retinal endothelial glycocalyx dimensions as well as a trend

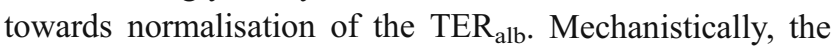
increased plasma hyaluronan levels and hyaluronidase levels in type 2 diabetes at baseline may reflect increased GAG catabolism in chronic hyperglycaemia [10], whereas sulodexide reduced GAG metabolism as attested to by a reduction of plasma hyaluronidase activity. Restoration of GAG metabolism has been suggested to improve the barrier function of the vasculature. Thus, Duling et al. previously showed that enhanced abundance of precursors for endothelial GAGs (particularly hyaluronan and unfractionated heparin) led to restoration of the vasculo-protective capacity of the endothelial glycocalyx $[12,29]$. In the present study, oral administration of sulodexide is expected to result in enhanced precursor abundance, which may drive the correction of the endothelial glycocalyx. As low-molecular-
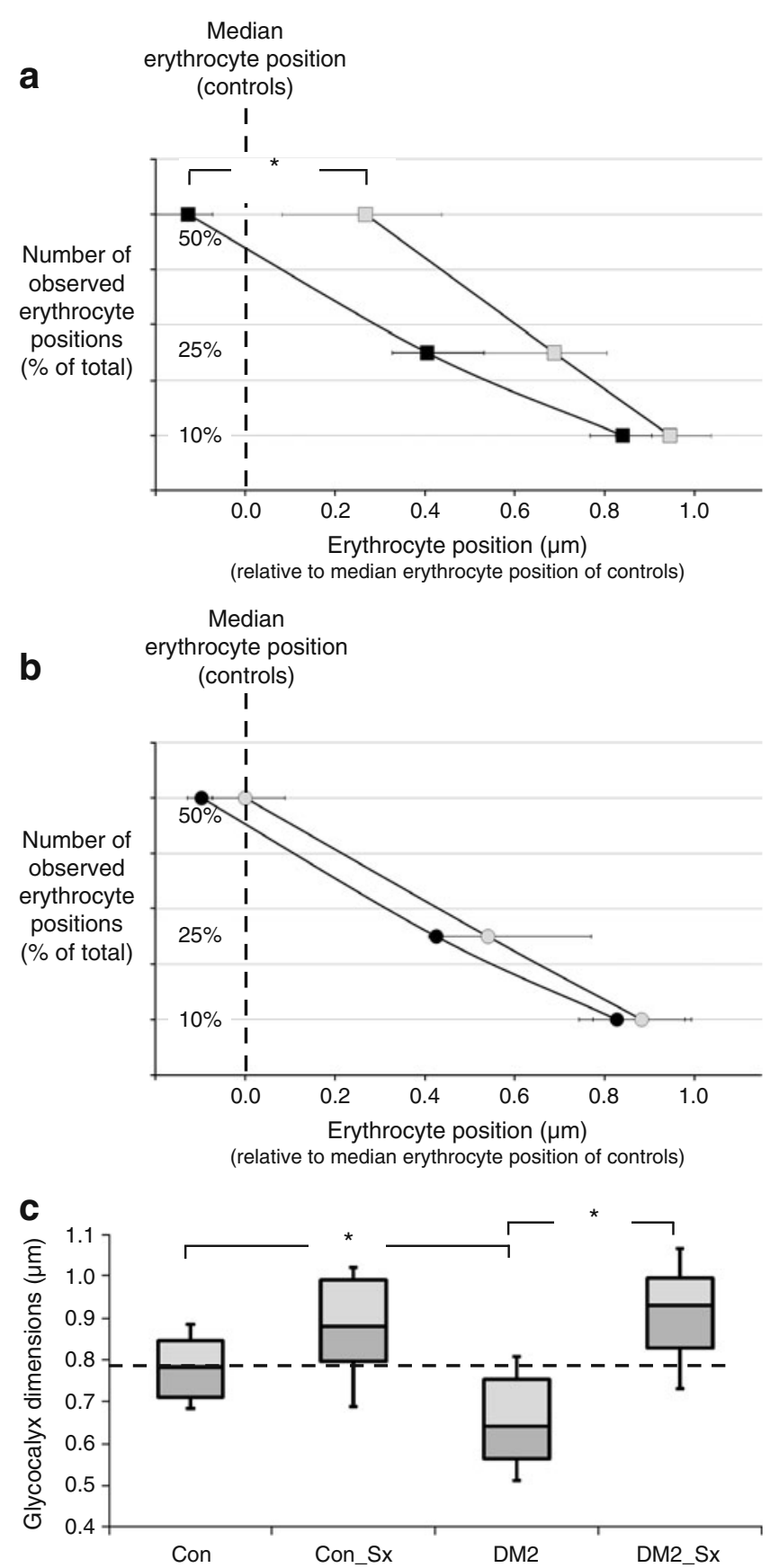

weight heparins may also exert beneficial effects on microalbuminuria and retinopathy in diabetes mellitus [30, 31], supplementation of other GAGs delivering comparable molar equivalents of glucosamine, galactosamine and glucuronic acid may share the beneficial properties of sulodexide for endothelial glycocalyx.

The apparent conflicting results regarding the effect of sulodexide on microalbuminuria deserve further discussion. The trials reporting a beneficial effect of sulodexide administration compared with placebo were all performed in renin-angiotensin-inhibitor-naive normotensive type 2 


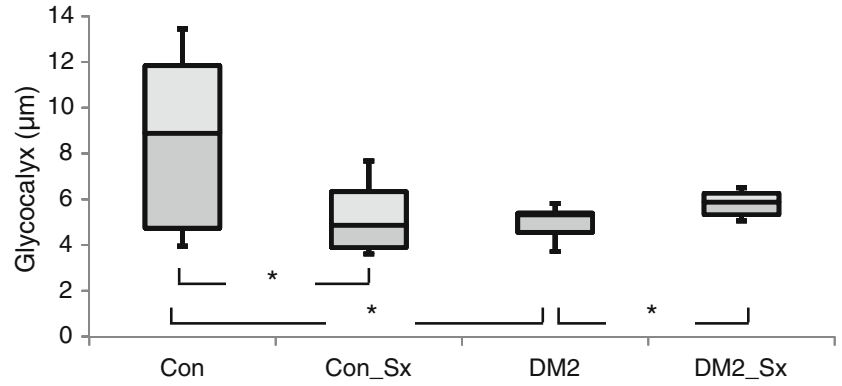

Fig. 4 The effect of sulodexide on retinal glycocalyx in control and diabetic participants. Glycocalyx dimensions in controls at baseline and after 8 weeks of treatment with sulodexide $(n=6$; con and Con_Sx, respectively) and diabetic participants ( $n=6$; DM2 and $\mathrm{DM}_{2}-\mathrm{Sx}$, respectively) measured by FAG/ICG. Individual glycocalyx dimensions were estimated by subtracting the intravascular distribution of two different fluorescent tracers: indocyanine green from fluorescein. Data are presented as whisker plots with median (interquartile range). ${ }^{*} p<0.05$

diabetes mellitus patients without overt vascular complications [32-34]. Recently, a beneficial effect of sulodexide was also reported that was additive to those of angiotensinconverting-enzyme inhibitors and/or angiotensin-receptor blockers (ARBs) [35]. Notably, type 2 diabetes mellitus patients in this trial were relatively young (mean age 55 years) without hypertension and with preserved renal function, and hence had less prolonged hyperglycaemic exposure. In contrast, two sulodexide trials performed in type 2 diabetes mellitus patients aiming to show protective effects of sulodexide additional to those of ARBs [36], were terminated prematurely because of lack of an effect on urinary albumin excretion [37]. However, patients included

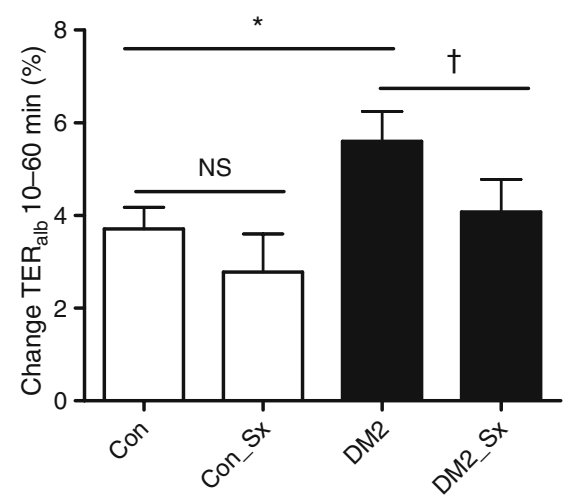

Fig. 5 The effect of sulodexide on vascular permeability in control and diabetic participants. Percentage change in TER $\mathrm{alb}_{\mathrm{al}}$ at baseline and after 8 weeks of treatment with sulodexide in controls $(n=10$; con and Con_Sx) and diabetic participants $(n=10 ; \mathrm{DM} 2$ and DM2_Sx); $p=\overline{\mathrm{NS}}$ for comparison of controls and controls following sulodexide treatment. Microvascular permeability was determined by the transcapillary escape rate of ${ }^{125}$ I-labelled albumin $\left(\mathrm{TER}_{\mathrm{alb}}\right)$ between 10 and $60 \mathrm{~min}$ after infusion. Data are presented as means \pm SD. ${ }^{*} p<0.05,{ }^{\dagger} p=0.08$
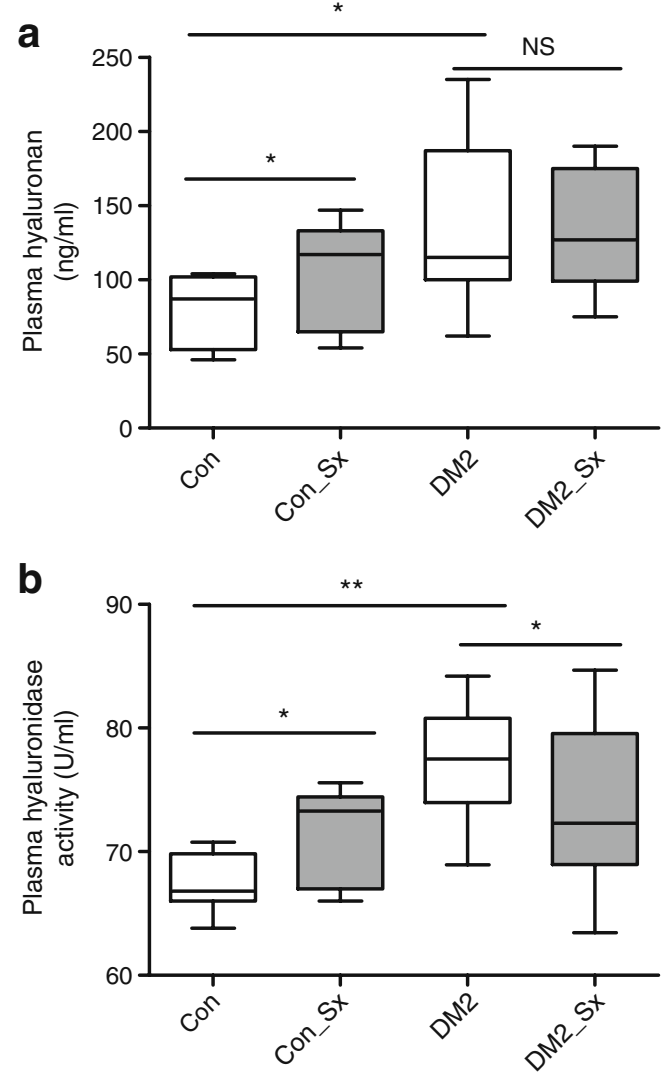

Fig. 6 The effect of sulodexide on plasma glycosaminoglycans in control and diabetic participants. a Circulating plasma hyaluronan levels at baseline and after 8 weeks of sulodexide administration in controls $(n=10$; con and Con_Sx, respectively) and diabetic participants ( $n=10$; DM2 and DM2_Sx, respectively). b Hyaluronidase levels at baseline and after 8 weeks of sulodexide administration in controls ( $n=10$; con and Con_Sx, respectively) and diabetic participants ( $n=10$; DM2 and DM2_Sx, respectively), $p<0.05$ for comparison of controls and controls following sulodexide treatment; $p<0.01$ for comparison of controls, controls following sulodexide treatment and diabetes group before sulodexide treatment; $p<0.05$ for diabetes group before and after sulodexide treatment. Data are presented as whisker plots with median (interquartile range). ${ }^{*} p<0.05, * * p<0.01$

in these trials were characterised by hypertension and microalbuminuria, indicating glomerular endothelial damage. Note that the microvascular endothelium may respond differently upon therapeutic intervention compared with glomerular endothelium (as determined by urinary albumin excretion), reflecting different pathogenic pathways [3840]. In this respect, urinary albumin excretion in diabetes mellitus has been attributed predominantly to proximal tubular dysfunction [41, 42], which is independent from changes in glycocalyx properties. Therefore, the lack of a change in urinary albumin excretion in the aforementioned trials does not preclude the finding of improved glycocalyx properties in our normoalbuminuric patients. In addition, the time scales may profoundly affect the observed results. Thus, vascular barrier function restoration may follow at a 
later stage when the endothelial glycocalyx is already restored [12, 13]. To definitively answer the question whether early intervention with sulodexide is able to prevent the development of diabetes-associated microvascular damage, a trial comparing sulodexide with placebo in newly diagnosed normotensive/normoalbuminuric patients with type 2 diabetes mellitus is warranted.

Mechanistically, measuring the impact of sulodexide on glycocalyx dimension and properties in health and disease is a challenge [15]. Endothelial glycocalyx thickness depends on the rate of synthesis, the rate of shedding as well as the circulating levels of GAGdegrading enzymes, including hyaluronidase and heparanase $[43,44]$. Surprisingly, an increase in the sublingual glycocalyx dimension with a concomitant decrease in retinal glycocalyx was observed following sulodexide administration in controls. These data imply that endothelial responses are heterogeneous not only in patients with type 2 diabetes compared with controls, but also in various vascular beds [45]. Apparently, the endothelial glycocalyx dimension is more closely regulated in the retina than the sublingual microvessels [46]. The driving force behind glycocalyx synthesis is most likely determined by enhanced abundance of the precursors glucosamine, galactosamine and glucuronic acid. Following intestinal degradation of orally administered sulodexide, increased levels of GAG precursors may contribute to the increased plasma levels of hyaluronan and hyaluronidase in controls. In diabetes mellitus, however, GAG degradation is increased [10, 21]. In these patients, decreased plasma hyaluronidase activity may be attributed to the inhibition of enzymatic glycocalyx degradation by the (sulodexide-mediated) increase in substrate bioavailability [12]. In support, sulodexide has been hypothesised to inhibit heparanase (and possibly hyaluronidase) activity, both of which are involved in GAG catabolism [47]. Therefore, in line with the pioneering study by Potter et al. [46], future in vitro endothelial glycocalyx studies will have to focus on the effect of GAG supplementation on the regulation of GAG-degrading enzymes in different vascular endothelia (glomerular, capillary and retina).

This study has several limitations. First, the dose of sulodexide used was not validated by a formal dosefinding study. Previous studies have indicated that a treatment period as long as 6 months may be required to establish the maximal effect [48]. This delayed mode of action virtually precludes performing a meaningful dosefinding study. Therefore, we selected the $200 \mathrm{mg}$ sulodexide dose based predominantly on previously published data [35]. These data have shown improvement in the endothelial barrier function using comparable concentrations of sulodexide in vitro [16]. Note that further dose escalation may also, at some point, be limited by the anticoagulant effects of heparan sulphates, which could potentially lead to bleeding complications. Second, our present proof-ofconcept study was rather small, with only type 2 diabetes patients without cardiovascular and/or proteinuric disease included. Whereas the findings are potentially interesting, they need further confirmation in a larger cohort as well as in type 2 diabetes patients with more severe stages of vascular complications.

In conclusion, individuals with type 2 diabetes mellitus are characterised by a clear perturbation of the endothelial glycocalyx layer, which is thought to reflect increased vascular vulnerability. Oral sulodexide administration improves endothelial glycocalyx dimension to the same extent in two different vascular beds (sublingual and retinal), most likely because of enhanced precursor abundance for GAG synthesis. Improvement of glycocalyx dimension coincided with a trend toward normalised systemic vascular permeability and GAG metabolism. Collectively, the present findings imply that restoration of endothelial glycocalyx in humans may be a promising target to attenuate vascular dysfunction in type 2 diabetes mellitus. Further research is required to evaluate which treatment modalities are most likely to establish improvement in endothelial glycocalyx dimension. More importantly, prospective studies should address whether the concept holds true that endothelial glycocalyx improvement predicts long-term cardiovascular benefit in diabetes mellitus.

Designations F. Holleman, M. Nieuwdorp, E.S.G. Stroes and H. Vink designed the study. L. N. Broekhuizen, B. A. Lemkes, H. L. Mooij, M. C. Meuwese and $\mathrm{H}$. Verberne performed the research. R. O. Schlingemann, contributed the analytical tools (FAG). L. N. Broekhuizen, B. A. Lemkes, M. Nieuwdorp, E. S. G. Stroes and H. Vink analysed the data and drafted the paper. All authors critically reviewed the manuscript.

Acknowledgements We gratefully acknowledge Alfa Wasserman for providing the sulodexide capsules. We acknowledge the Dutch Heart Foundation (grant number 2006B088) for their financial support. We gratefully acknowledge J. Jong and J. Sierts for excellent laboratory assistance. This study was funded by an unrestricted Novartis Foundation for Cardiovascular Excellence grant (2006) to M. Nieuwdorp/E. S. G. Stroes, Dutch Heart Foundation (grant number 2005T037). M. Nieuwdorp is currently supported by a ZONMW/ NWO VENI grant 2008 (016.096.044).

Duality of interest All authors have no conflict of interest to declare. Alfa Wasserman provided the sulodexide, but was not involved in data analyses/interpretation or writing of the manuscript.

Open Access This article is distributed under the terms of the Creative Commons Attribution Noncommercial License which permits any noncommercial use, distribution, and reproduction in any medium, provided the original author(s) and source are credited. 


\section{References}

1. Deckert T, Feldt-Rasmussen B, Borch-Johnsen K, Jensen T, Kofoed-Enevoldsen A (1989) Albuminuria reflects widespread vascular damage. The Steno hypothesis. Diabetologia 32:219-226

2. Gerstein HC, Mann JF, Yi Q et al (2001) Albuminuria and risk of cardiovascular events, death, and heart failure in diabetic and nondiabetic individuals. JAMA 25(286):421-426

3. Morrish NJ, Wang SL, Stevens LK, Fuller JH, Keen H (2001) Mortality and causes of death in the WHO multinational study of vascular disease in diabetes. Diabetologia 44(Suppl 2):S14-S21

4. Brownlee M (2001) Biochemistry and molecular cell biology of diabetic complications. Nature 414:813-820

5. Nieuwdorp M, van Haeften TW, Gouverneur MC et al (2006) Loss of endothelial glycocalyx during acute hyperglycaemia coincides with endothelial dysfunction and coagulation activation in vivo. Diabetes 55:480-486

6. van Haaren PM, VanBavel E, Vink H, Spaan JA (2003) Localization of the permeability barrier to solutes in isolated arteries by confocal microscopy. Am J Physiol Heart Circ Physiol 285:H2848-H2856

7. Chappell D, Jacob M, Paul O et al (2009) The glycocalyx of the human umbilical vein endothelial cell: an impressive structure ex vivo but not in culture. Circ Res 104:1313-1317

8. Chappell D, Jacob M, Rehm M et al (2008) Heparinase selectively sheds heparan sulphate from the endothelial glycocalyx. Biol Chem 389:79-82

9. Pries AR, Secomb TW, Gaehtgens P (2000) The endothelial surface layer. Pflugers Arch 440:653-666

10. Nieuwdorp M, Mooij HL, Kroon J et al (2006) Endothelial glycocalyx damage coincides with microalbuminuria in type 1 diabetes. Diabetes 55:1127-1132

11. Constantinescu AA, Vink H, Spaan JA (2003) Endothelial cell glycocalyx modulates immobilization of leukocytes at the endothelial surface. Arterioscler Thromb Vasc Biol 23:15411547

12. Henry CB, Duling BR (1999) Permeation of the luminal capillary glycocalyx is determined by hyaluronan. Am J Physiol 277: H508-H514

13. Rossetti L, Hawkins M, Chen W, Gindi J, Barzilai N (1995) In vivo glucosamine infusion induces insulin resistance in normoglycemic but not in hyperglycemic conscious rats. J Clin Invest 96:132-140

14. Buse MG (2006) Hexosamines, insulin resistance, and the complications of diabetes: current status. Am J Physiol Endocrinol Metab 290:E1-E8

15. Weiss R, Niecestro R, Raz I (2007) The role of sulodexide in the treatment of diabetic nephropathy. Drugs 67:2681-2696

16. Ciszewicz M, Polubinska A, Antoniewicz A, Suminska-Jasinska K, Breborowicz A (2009) Sulodexide suppresses inflammation in human endothelial cells and prevents glucose cytotoxicity. Transl Res 153:118-123

17. Nieuwdorp M, Meuwese MC, Mooij HL et al (2008) Measuring endothelial glycocalyx dimensions in humans: a potential novel tool to monitor vascular vulnerability. J Appl Physiol 104:845-852

18. Yoneya S (2004) A new approach for studying the retinal and choroidal circulation. Nippon Ganka Gakkai Zasshi 108:836-861 (article in Japanese)

19. Vink H, Duling BR (1996) Identification of distinct luminal domains for macromolecules, erythrocytes, and leukocytes within mammalian capillaries. Circ Res 79:581-589

20. van Eijk LT, Pickkers P, Smits P, van den Broek W, Bouw MP, van der Hoeven JG (2005) Microvascular permeability during experimental human endotoxemia: an open intervention study. Crit Care 9:R157-R164
21. Nieuwdorp M, Holleman F et al (2007) Perturbation of hyaluronan metabolism predisposes patients with type 1 diabetes mellitus to atherosclerosis. Diabetologia 50:12881293

22. Chappell D, Jacob M, Hofmann-Kiefer K et al (2009) Antithrombin reduces shedding of the endothelial glycocalyx following ischaemia/reperfusion. Cardiovasc Res 83:388-396

23. van den Berg BM, Vink H, Spaan JA (2003) The endothelial glycocalyx protects against myocardial edema. Circ Res 92:592594

24. Mehta D, Malik AB (2006) Signaling mechanisms regulating endothelial permeability. Physiol Rev 86:279-367

25. Perrin RM, Harper SJ, Bates DO (2007) A role for the endothelial glycocalyx in regulating microvascular permeability in diabetes mellitus. Cell Biochem Biophys 49:65-72

26. Jeansson M, Haraldsson B (2006) Morphological and functional evidence for an important role of the endothelial cell glycocalyx in the glomerular barrier. Am J Physiol Renal Physiol 290:F111F116

27. Singh A, Satchell SC, Neal CR, McKenzie EA, Tooke JE, Mathieson PW (2007) Glomerular endothelial glycocalyx constitutes a barrier to protein permeability. J Am Soc Nephrol 18:2885-2893

28. Russo LM, Sandoval RM, Campos SB, Molitoris BA, Comper WD, Brown D (2009) Impaired tubular uptake explains albuminuria in early diabetic nephropathy. J Am Soc Nephrol 20:489-494

29. Rubio-Gayosso I, Platts SH, Duling BR (2006) Reactive oxygen species mediate modification of glycocalyx during ischemiareperfusion injury. Am J Physiol Heart Circ Physiol 290:H2247$\mathrm{H} 2256$

30. Tamsma JT, van der Woude FJ, Lemkes HH (1996) Effect of sulphated glycosaminoglycans on albuminuria in patients with overt diabetic nephropathy. Nephrol Dial Transplant 11:182185

31. van der Pijl JW, van der Woude FJ, Swart W, van Es LA, Lemkes $\mathrm{HH}$ (1997) Effect of danaparoid sodium on hard exudates in diabetic retinopathy. Lancet 350:1743-1745

32. Dedov I, Shestakova M, Vorontzov A, Palazzini E (1997) A randomised, controlled study of sulodexide therapy for the treatment of diabetic nephropathy. Nephrol Dial Transplant $12: 2295-2300$

33. Solini A, Vergnani L, Ricci F, Crepaldi G (1997) Glycosaminoglycans delay the progression of nephropathy in NIDDM. Diab Care 20:819-823

34. Gambaro G, Kinalska I, Oksa A et al (2002) Oral sulodexide reduces albuminuria in microalbuminuric and macroalbuminuric type 1 and type 2 diabetic patients: the Di.N.A.S. randomized trial. J Am Soc Nephrol 13:1615-1625

35. Heerspink HL, Greene T, Lewis JB et al (2008) Effects of sulodexide in patients with type 2 diabetes and persistent albuminuria. Nephrol Dial Transplant 23:1946-1954

36. Lambers Heerspink HJ, Fowler MJ, Volgi J, Collaborative Study Group et al (2007) Rationale for and study design of the sulodexide trials in type 2 diabetic, hypertensive patients with microalbuminuria or overt nephropathy. Diabet Med 24:12901295

37. www.clinicaltrials.gov identifier: NCT00130312 and NCT003 42238

38. Ceol M, Gambaro G, Sauer U, Baggio B, Anglani F, Forino F (2000) Glycosaminoglycan therapy prevents TGF- $\beta 1$ overexpression and pathologic changes in renal tissue of long-term diabetic rats. J Am Soc Nephrol 11:2324-2336

39. Higashi S, Clermont AC, Dhir V, Bursell SE (1998) Reversibility of retinal flow abnormalities is disease-duration dependent in diabetic rats. Diabetes 47:653-659 
40. Nosadini R, Velussi M, Brocco E (2005) Altered transcapillary escape of albumin and microalbuminuria reflects two different pathogenetic mechanisms. Diabetes 54:228-233

41. Lauer ME, Hascall VC, Wang A (2007) Heparan sulfate analysis from diabetic rat glomeruli. J Biol Chem 282:843-852

42. Wang A, Hascall VC (2004) Hyaluronan structures synthesized by rat mesangial cells in response to hyperglycaemia induce monocyte adhesion. J Biol Chem 279:10279-10285

43. Ikegami-Kawai M, Suzuki A, Karita I, Takahashi T (2003) Increased hyaluronidase activity in the kidney of streptozotocininduced diabetic rats. J Biochem 134:875-880

44. Maxhimer JB, Somenek M, Rao G, Pesce CE, Baldwin D Jr, Gattuso $P$ (2005) Heparanase-1 gene expression and regulation by high glucose in renal epithelial cells: a potential role in the pathogenesis of proteinuria in diabetic patients. Diabetes 54:2172-2178

45. Aird WC (2003) Endothelial cell heterogeneity. Crit Care Med 31 (Suppl):S221-S230

46. Potter DR, Jiang J, Damiano ER (2009) The recovery time course of the endothelial cell glycocalyx in vivo and its implications in vitro. Circ Res 104:1318-1325

47. Wijnhoven TJ, Lensen JF, Rops AL et al (2007) Anti-proteinuric effects of glycosaminoglycan-based drugs. Curr Opin Mol Ther 9:364-377

48. Harenberg J (1998) Review of pharmacodynamics, pharmacokinetics, and therapeutic properties of sulodexide. Med Res Rev $18: 1-20$ 